Network Working Group

Request for Comments: 4496

Category: Informational
M. Stecher Secure Computing

A. Barbir Nortel

May 2006

\title{
Open Pluggable Edge Services (OPES) SMTP Use Cases
}

\section{Status of This Memo}

This memo provides information for the Internet community. It does not specify an Internet standard of any kind. Distribution of this memo is unlimited.

Copyright Notice

Copyright (C) The Internet Society (2006).

\section{Abstract}

The Open Pluggable Edge Services (OPES) framework is application agnostic. Application-specific adaptations extend that framework. This document describes OPES SMTP use cases and deployment scenarios in preparation for SMTP adaptation with OPES.

Table of Contents

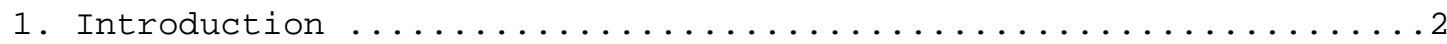

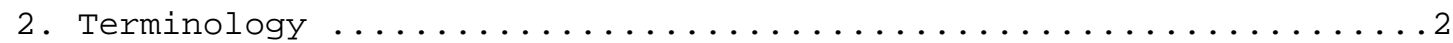

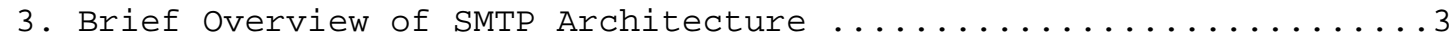

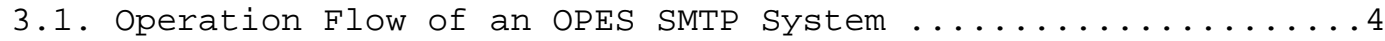

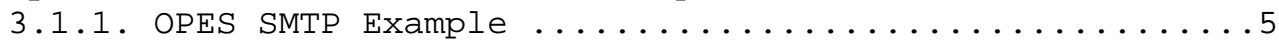

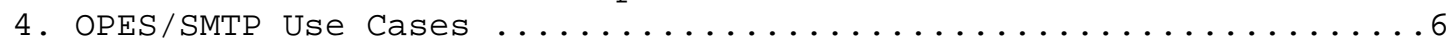
4.1. Security Filters Applied to Email Messages ..........6

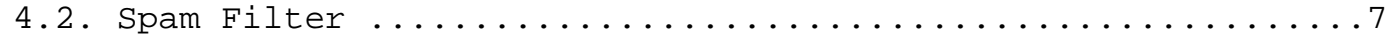

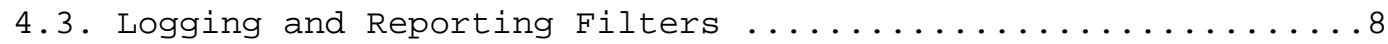

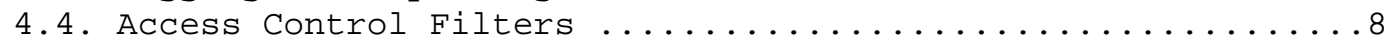

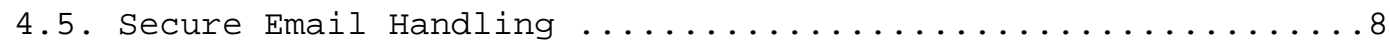

4.6. Email Format Normalization ....................

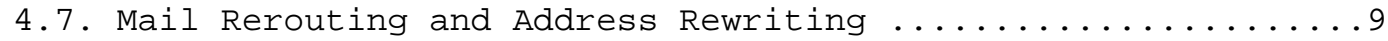

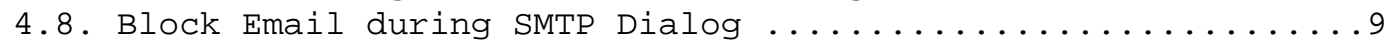

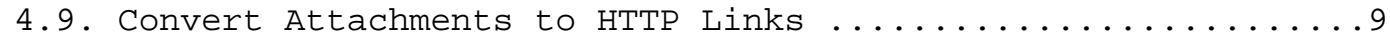

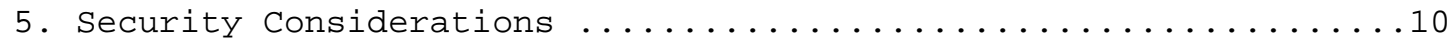

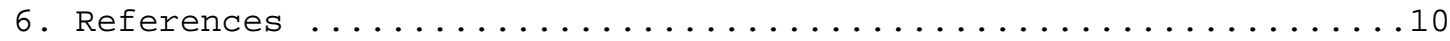

6.1. Normative References .........................10

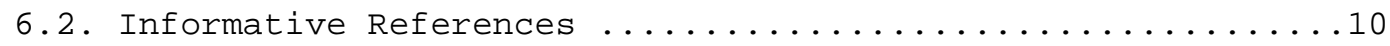

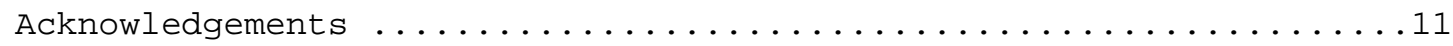




\section{Introduction}

The Open Pluggable Edge Services (OPES) architecture [1] enables cooperative application services (OPES services) between a data provider, a data consumer, and zero or more opes processors. The application services under consideration analyze and possibly transform application-level messages exchanged between the data provider and the data consumer. The OPES processor can distribute the responsibility of service execution by communicating and collaborating with one or more remote callout servers.

The execution of such services is governed by a set of rules installed on the OPES processor. The rule evaluation can trigger the execution of service applications local to the opEs processor or on a remote callout server.

Use cases for OPES based on HTTP [8] are described in [2]. This work focuses on OPES for SMTP [7] use cases, whereby additional use cases and enhancements to the types of OPES services defined in [2] are provided.

In SMTP, the OPES processor may be any agent participating in SMTP exchanges, including a Mail Submission Agent (MSA), a Mail Transfer Agent (MTA), a Mail Delivery Agent (MDA), and a Mail User Agent (MUA). This document focuses on use cases in which the OPES processor is a MTA.

SMTP is a store-and-forward protocol. Current email filtering systems either operate during the SMTP exchange or on messages that have already been received, after the SMTP connection has been closed (for example, in an $\mathrm{MTA}^{\prime} \mathrm{s}$ message queue).

This work focuses on SMTP-based services that want to modify command values or want to block SMTP commands. In order to block a command, the service will provide an error message that the MTA should use in response to the command it received. An OPES MTA will be involved in SMTP command modification and command satisfaction, analogous to request modification and request satisfaction from HTTP [8] .

2. Terminology

The key words "MUST", "MUST NOT", "REQUIRED", "SHALL", "SHALL NOT", "SHOULD", "SHOULD NOT", "RECOMMENDED", "MAY", and "OPTIONAL" in this document are to be interpreted as described in [6]. When used with the normative meanings, these key words will be all uppercase. Occurrences of these words in lowercase comprise normal prose usage, with no normative implications. 


\section{Brief Overview of SMTP Architecture}

The SMTP design, taken from RFC 2821 [7], is shown in Figure 1. When an SMTP client has a message to transmit, it establishes a two-way transmission channel to an SMTP server. The responsibility of an SMTP client is to transfer mail messages to one or more SMTP servers, or report its failure to do so.

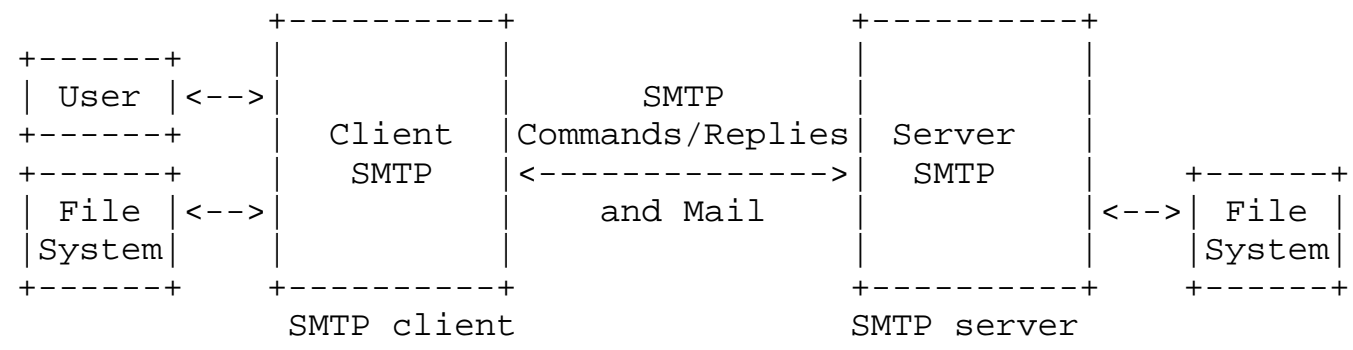

Figure 1: SMTP Design

In some cases, the domain name(s) transferred to, or determined by, an SMTP client will identify the final destination(s) of the mail message. In other cases, the domain name determined will identify an intermediate destination through which those mail messages are to be relayed.

An SMTP server may be either the ultimate destination or an intermediate "relay" or "gateway" (that is, it may transport the message further using some protocol other than SMTP or using again SMTP and then acting as an SMTP client).

SMTP commands are generated by the SMTP client and sent to the SMTP server. SMTP responses are sent from the SMTP server to the SMTP client in response to the commands. SMTP message transfer can occur in a single connection between the original SMTP sender and the final SMTP recipient, or it can occur in a series of hops through intermediary systems. SMTP clients and servers exchange commands and responses and eventually the mail message body.

Figure 2 expands on the mail flow in an SMTP system. Further information about the architecture of email in the Internet may be found in [9]. 


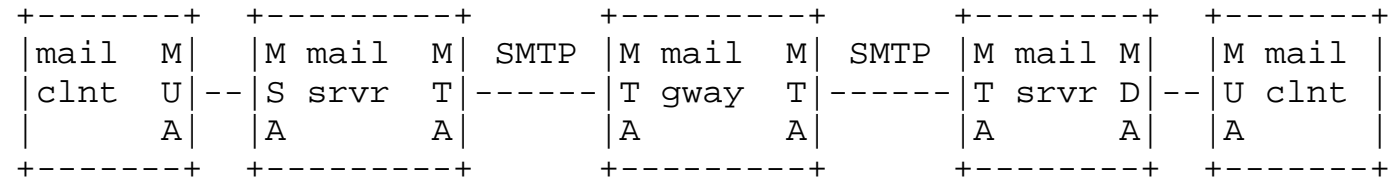

Figure 2: Expanded SMTP Flow

In this work, the OPES processor may be any agent that is participating in SMTP exchanges, including an MSA, MTA, MDA, and MUA. However, this document focuses on use cases in which the OPES processor uses the SMTP protocol or one of the protocols derived from SMTP Message Submission (SUBMIT) [10] and the Local Mail Transfer Protocol (LMTP) [11]).

\subsection{Operation Flow of an OPES SMTP System}

In this work, an MTA is the OPES processor device that sits in the data stream of the SMTP protocol. The OPES processor gets enhanced by an OCP (OPES callout protocol) [3] client that allows it to vector out data to the callout server. The filtering functionality is on the callout server.

A client (a mail user) starts with an email client program (MUA) . The user sends email to an outgoing email server. In the email server, there is an MSA (mail submission agent) that is waiting to receive email from the user. The MSA uses an MTA within the same server to forward the user email to other domains. (Communication between the MUA and MSA may be via SMTP, SUBMIT [10], or something else such as MAPI).

The MTA in the user email server may directly contact the email server of the recipient or may use other intermediate email gateways. The sending email server and all intermediate gateway MTAs usually communicate using SMTP. Communication with the destination email server usually uses SMTP or its derivative, LMTP [11].

In the destination email server, a mail delivery agent (MDA) may deliver the email to the recipient's mailbox. The email client program of the recipient might use a different protocol (such as the Post Office Protocol version 3 (POP3) or IMAP) to access the mailbox and retrieve/read the messages. 


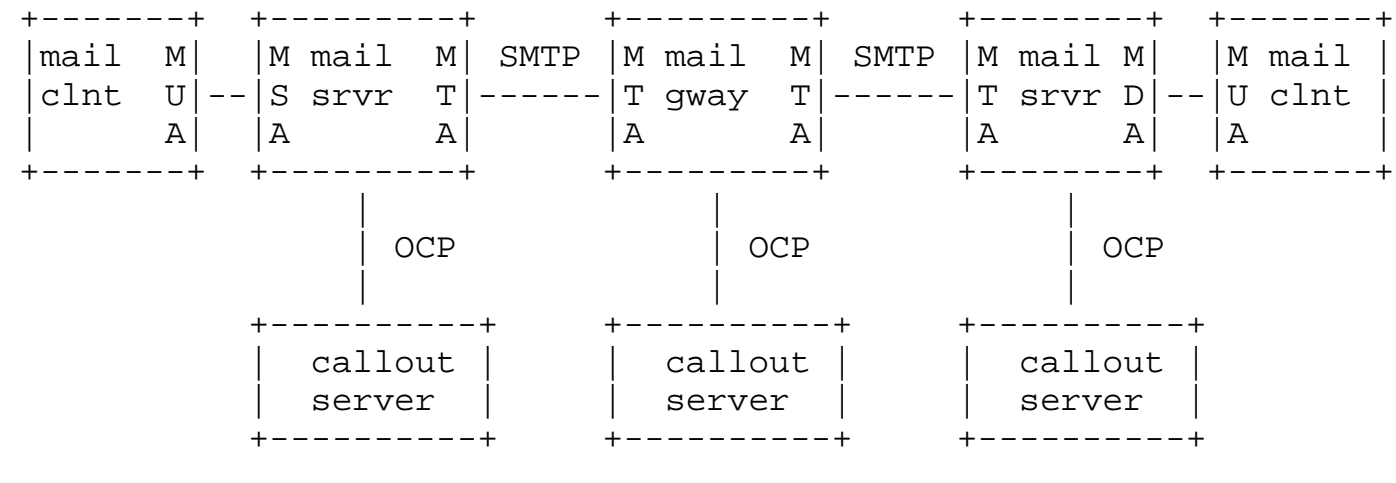

Figure 3: OPES SMTP Flow

From Figure 3, the MTA (the OPES processor) is either receiving or sending an email (or both) within an email server/gateway. An OPES processor might be the sender's SMTP server, the destination SMTP server, or any intermediate SMTP gateway. (Which building block belongs to which authoritative domain is an important question but different from deployment to deployment.) Note that this figure shows multiple OPES deployment options in a typical chain of mail servers and gateways with different roles as MSA, MTA, and MDA; the OPES standard case, however, will only have a single OPES processor and a single callout server in the message flow.

\subsubsection{OPES SMTP Example}

A typical (minimum) SMTP dialog between two OPES SMTP processors (MTA) will consist of the following (C: means client, $S$ : means server):

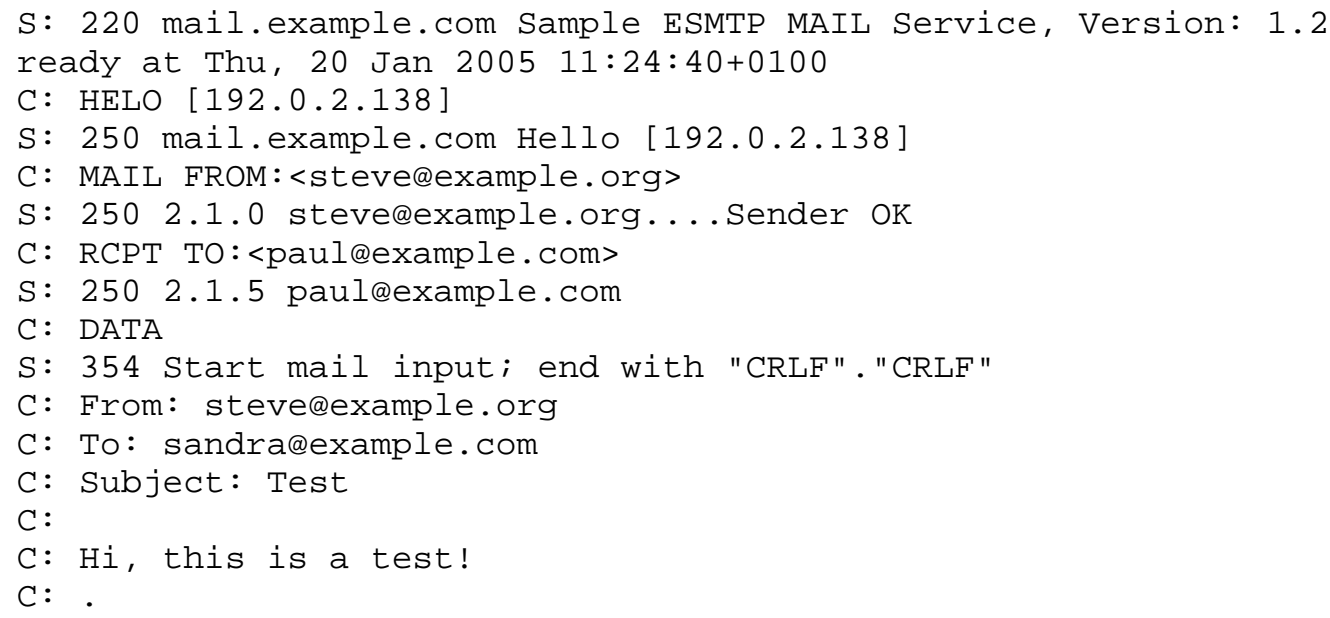


S: 250 2.6.0 "MAILOm4b1f@mail.example.com" Queued mail for delivery

C: QUIT

S: 221 2.0.0 mail.example.com Service closing transmission channel

The client (C:) is issuing SMTP commands and the server (S:) is generating responses. All responses start with a status code and then some text. At minimum, 4 commands are needed to send an email. Together, all commands and responses to send a single email message form "the dialog". The mail message body is the data sent after the "DATA" command. An OPES processor could see that as command modification.

If a callout service wants to adapt the email message body, it is mainly interested in this part of the dialog:

From: steve@example.org

To: sandra@example.com

Subject: Test

Hi, this is a test!

The callout service may need to examine values of previous commands of the same dialog. For example, the callout service needs to examine the value of the RCPT command (it is "pauldexample.com"), which is different from the "sandradexample.com" that the email client displays in the visible "To" field. That might be an important fact for some filters such as spam filters (Section 4.2).

4. OPES/SMTP Use Cases

In principle, all filtering that is deployed at SMTP gateways today and tomorrow defines use cases for OPES callout filtering. An OCP/SMTP callout protocol will enable an SMTP gateway to vector out (parts of) an SMTP message or parts of the SMTP dialog to a callout server that is then performing actions on behalf of the gateway. (OCP/SMTP would be a profile defined for OCP analogous to the OCP/HTTP profile [4] that has been defined earlier.)

Here is a collection of some typical use cases describing different filtering areas and different actions caused by those filters.

\subsection{Security Filters Applied to Email Messages}

These filters concentrate on the email message body and usually filter the email sections one by one. Email sections (attachments) that violate the security policy (e.g., because they contain a virus 
or contain an unwanted mime type) define an event that can cause a combination of different actions to be performed:

o The attachment is replaced by an error message.

- The email is marked by inserting a warning into the subject or the email body.

- An additional header is added for post-processing steps.

- The email storage is advised to put the email into quarantine.

- Notifications are sent to sender, recipients, and/or administrators.

- The incident is reported to other tools such as intrusion detection applications.

These kinds of filters usually do not require working with elements of the SMTP dialog other than the email message body. An exception to this is the need to map email senders and recipients to different security sub-policies that are used for a particular message. A security filter may therefore require receiving the information of the RCPT TO and MAIL FROM commands as meta data with the email message body it examines.

\subsection{Spam Filter}

Next to security filters, spam filters are probably the most wanted filtering application today. Spam filters use several methods. They concentrate most on the email message body (that also includes the email headers), but many of these filters are also interested in the values of the other SMTP commands in order to compare the SMTP sender/recipients with the visible From/To fields. They may even want to get the source IP of the connected SMTP client as meta information to verify this against lists of open relays, known spammers, etc.

These are typical actions that could be performed when a message has been classified as spam:

o Add a mark to the subject of the email.

- Add an additional header for post-processing steps.

- The email storage is advised to put the email into a spam queue.

o The email is rejected or returned to the sender. 


\subsection{Logging and Reporting Filters}

The nature of these kinds of filters is not to modify the email message. Depending on what is being logged or reported on, the filter may need access to any part of the SMTP dialog. Most wanted is the sender and recipient information. Depending on the ability of the OPES processor to pre-calculate and transfer information about the message body, the callout filter may want to see the email message body itself or just that meta info; an example is the email size. This information would be typical logging and reporting information that is easy for the SMTP gateway to calculate although not a direct parameter of the SMTP dialog. Transferring the complete email message body only because the callout server wants to calculate its size would be a waste of network resources.

\subsection{Access Control Filters}

These filters operate on the values of the MAIL FROM and RCPT TO commands of the SMTP dialog. They run an access control policy to determine whether a sender is currently allowed to send a message to the given recipients. The values of HELO/EHLO, AUTH, and STARTTLS commands may also be applied. The result of this filter has a direct influence on the SMTP response that the OPES processor has to send to its peer for the filtered SMTP command.

\subsection{Secure Email Handling}

Filters of this kind can support an email gateway to centrally encode and decode email, and to set and to verify email signatures. They will therefore modify the email message body to encrypt, decrypt, verify, or sign the message, or use an action as specified in the "Security Filter" (Section 4.1) section if the decryption or signature verification fails.

Sending the SMTP sender and recipient information as meta data to these filters is mission critical because these filters may not trust the information found in the header section of the email message body.

\subsection{Email Format Normalization}

SMTP messages may be sent with an illegal or uncommon format; this may have happened by a buggy SMTP application or on purpose in order to exploit vulnerabilities of other products. A normalization filter can correct the email format. The format correction can be done for the email body and for the value of other SMTP commands. An example for the email body format correction would be a strange length of UUencoded lines or unusual names of MIME sections. Command values 
may be analysed against buffer overflow exploits; a rewrite will not always be possible in this case (cannot simply rewrite an email address that is very long) but will require that the callout server tells the OPES processor to send an error response in reply to such a command.

\subsection{Mail Rerouting and Address Rewriting}

A corporation with multiple locations may want to deploy a central gateway that receives all email messages for all employees and then determines at which location the mail storage of the employee resides. The callout server will then need the RCPT TO command value and it will look up the location in the corporate directory service. It then tells either the OPES processor where the next SMTP server is (i.e., the next SMTP server to connect to) or it rewrites the recipient address; in the first case, the SMTP servers at the different locations accept emails of the same domain as the central gateway does; in the second case, the other locations will probably use the sublocation of the original domain (joedexample.org -> joedfr.example.org or joedde.example.org).

\subsection{Block Email during SMTP Dialog}

In a first step, the callout server will check the sender and recipient information that was transmitted in the SMTP dialog; that information again maps to a policy that will deny all messages either from that sender or to that recipient, or it checks the body of the email and classifies it (maybe just by looking for some words in the subject or by doing in-depth content analysis), which can then also lead to the decision to deny the message.

Unlike previous examples, this use case wants to deny the email while the SMTP dialog is still active, i.e., before the OPES processor finally accepted the message. Depending on the exact policy, the error response should then be sent in reply to the MAIL FROM, RCPT TO, or DATA command.

\subsection{Convert Attachments to HTTP Links}

This use case will only modify the email message body without any other influence on the SMTP dialogs, mail routing, etc. Larger sections (attachments) are removed from the email, and the content is stored on a web server. A link to that new URL is then added into the text of a first section that is likely to be displayed by an email client. Storing the attachments onto the web server is not in the scope of the OPES/SMTP scenario and needs to be implemented by the callout server. 


\section{Security Considerations}

Application-independent security considerations are documented in application-agnostic OPES specifications [5]. This document contains only use cases and defines no protocol operations. Security considerations for protocols that appear in these use cases are documented in the corresponding protocol specifications.

Use case "Secure Email Handling" (Section 4.5) is special in this regard because it requires the extension of the end-to-end encryption model and a secure handling of private cryptographic keys when creating digital signatures or when decrypting messages. Both are out of scope of OPES protocol specifications. An implementation of such a service raises security issues (such as availability and storage of cryptographic keys) that must be addressed regardless of whether the implementation happens within an MTA or within an OPES callout server.

6. References

6.1. Normative References

[1] Barbir, A., Penno, R., Chen, R., Hofmann, M., and H. Orman, "An Architecture for Open Pluggable Edge Services (OPES)", RFC 3835, August 2004 .

[2] Barbir, A., Burger, E., Chen, R., McHenry, S., Orman, H., and R. Penno, "Open Pluggable Edge Services (OPES) Use Cases and Deployment Scenarios", RFC 3752, April 2004.

[3] Rousskov, A., "Open Pluggable Edge Services (OPES) Callout Protocol (OCP) Core", RFC 4037, March 2005.

[4] Rousskov, A. and M. Stecher, "HTTP Adaptation with Open Pluggable Edge Services (OPES)", RFC 4236, November 2005.

[5] Barbir, A., Batuner, O., Srinivas, B., Hofmann, M., and H. Orman, "Security Threats and Risks for Open Pluggable Edge Services (OPES)", RFC 3837, August 2004.

[6] Bradner, S., "Key words for use in RFCs to Indicate Requirement Levels", BCP 14, RFC 2119, March 1997.

6.2. Informative References

[7] Klensin, J., "Simple Mail Transfer Protocol", RFC 2821, April 2001 . 
[8] Fielding, R., Gettys, J., Mogul, J., Frystyk, H., Masinter, L., Leach, P., and T. Berners-Lee, "Hypertext Transfer Protocol -HTTP/1.1", RFC 2616, June 1999.

[9] Crocker, D., "Internet Mail Architecture", Work in Progress, March 2005 .

[10] Gellens, R. and J. Klensin, "Message Submission", RFC 2476, December 1998 .

[11] Myers, J., "Local Mail Transfer Protocol", RFC 2033, October 1996.

Acknowledgements

Many thanks to everybody who provided input for the use case examples, namely, jfc and Markus Hofmann. Thanks also for the discussion and feedback given on the OPES mailing list.

Authors' Addresses

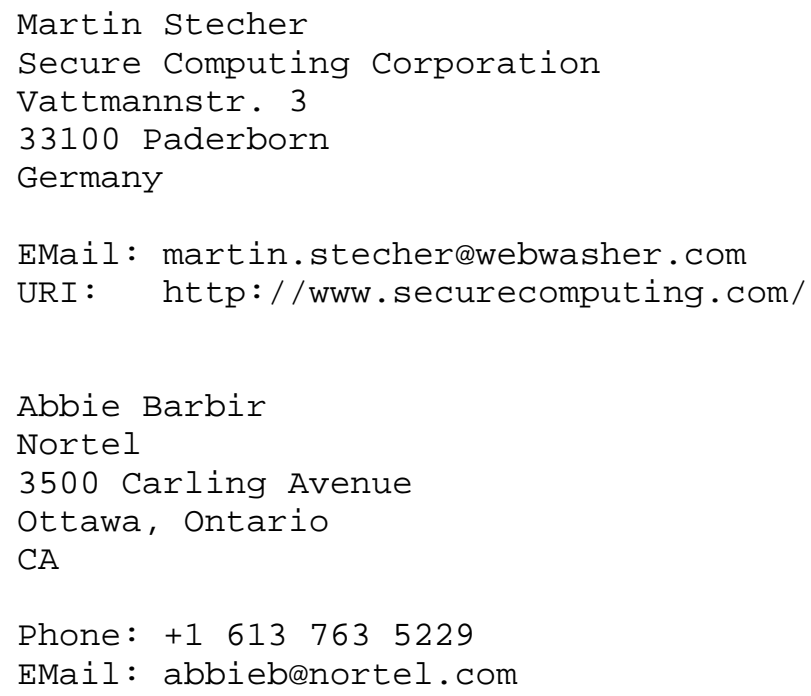


Full Copyright statement

Copyright (C) The Internet Society (2006).

This document is subject to the rights, licenses and restrictions contained in BCP 78, and except as set forth therein, the authors retain all their rights.

This document and the information contained herein are provided on an "AS IS" basis and THE CONTRIBUTOR, THE ORGANIZATION HE/SHE REPRESENTS OR IS SPONSORED BY (IF ANY), THE INTERNET SOCIETY AND THE INTERNET ENGINEERING TASK FORCE DISCLAIM ALL WARRANTIES, EXPRESS OR IMPLIED, INCLUDING BUT NOT LIMITED TO ANY WARRANTY THAT THE USE OF THE INFORMATION HEREIN WILL NOT INFRINGE ANY RIGHTS OR ANY IMPLIED WARRANTIES OF MERCHANTABILITY OR FITNESS FOR A PARTICULAR PURPOSE.

Intellectual Property

The IETF takes no position regarding the validity or scope of any Intellectual property Rights or other rights that might be claimed to pertain to the implementation or use of the technology described in this document or the extent to which any license under such rights might or might not be available; nor does it represent that it has made any independent effort to identify any such rights. Information on the procedures with respect to rights in RFC documents can be found in BCP 78 and BCP 79 .

Copies of IPR disclosures made to the IETF Secretariat and any assurances of licenses to be made available, or the result of an attempt made to obtain a general license or permission for the use of such proprietary rights by implementers or users of this specification can be obtained from the IETF on-line IPR repository at http://www.ietf.org/ipr.

The IETF invites any interested party to bring to its attention any copyrights, patents or patent applications, or other proprietary rights that may cover technology that may be required to implement this standard. Please address the information to the IETF at ietf-ipreietf.org.

Acknowledgement

Funding for the RFC Editor function is provided by the IETF Administrative Support Activity (IASA) . 